\title{
The Nature of UV-selected Galaxies in the Chandra Deep Field South
}

\author{
D.F. de Mello, J.P. Gardner \\ Laboratory for Astronomy and Solar Physics, Code 681, Goddard Space \\ Flight Center, Greenbelt, MD 20771, USA \\ T. Dahlen ${ }^{1}$, C.J. Conselice ${ }^{2}$, N.A. Grogin ${ }^{1}$, A.M Koekemoer ${ }^{1}$ \\ ${ }^{1}$ Space Telescope Science Institute, USA ${ }^{2}$ Caltech, USA
}

\begin{abstract}
.
We report the first results of the ongoing survey using HST/WFPC2 (F300W) in parallel with ACS within the Chandra Deep Field South. A sample of 34 objects were identified in the WFPC2 images and their counterparts were found in the ACS images taken by the GOODS team; 6 of them are stars. Galaxies were classified as early-, late-types, and starbursts by template fitting which was also used to determine their photometric redshifts $(\mathrm{z}<1)$. Analysis of the light concentration, asymmetry and clumpiness shows that this sample is a mixed bag, containing dwarf ellipticals, early- and late-spirals, and peculiar objects which resemble mergers in progress. This result has important implications for galaxy evolution since the intermediate redshifts are the epoch when the rise in the volume-averaged star formation rate occurs.
\end{abstract}

\section{The UV-selected Sample}

In this contribution we report the first results of a survey using the HST/WFPC2 (F300W) in combination with the HST/ACS multi-wavelength images. UV observations probe unobscured star formation, therefore, observing in the UV directly accesses the population of objects that contributes to the 'observed' rise in the star-formation rate at intermediate-z (Madau et al. 1996). For parallel fields that fell within the Great Observatories Origins Deep Survey (GOODS) fields, we took near-UV images with the F300W filter (U band) of galaxies that have been imaged at redder wavelengths with ACS. We detected 58 objects on the U-band image using SExtractor v2.2.2 (Bertin \& Arnouts 1996, hereafter $\mathrm{SE})$ in 8 WFPC2 images of the Chandra Deep Field South. Their magnitude range is $18<\mathrm{m}_{U}<26$ where $\mathrm{m}_{U}$ (AB system) is SE's mag_auto. These numbers agree with what is expected with the $U$ band ( 6 objects in 2 to 3 -orbit exposure). For comparison, the HDF project included a long (45 orbit) U exposure and has 133 objects with $23.5<\mathrm{m}_{U}<25$ (Vega system). The next step was to match the U-band catalog with the ACS B-band catalog $\left(\mathrm{B}_{435}\right)$ produced by the GOODS team (Giavalisco et al. 2004). We adopted a maximum offset radius of 1.5 arcsec between the WFPC2 coordinates and the ACS B band, and identified a total 
of 34 objects; 6 of them were identified as stars. Photometric redshifts were estimated by the GOODS team (Mobasher et al. 2004). The majority (26 out of 28) of the objects in the U-band sample has $\mathrm{z}<1$.

\section{Results}

Template fitting obtained from the photometric-z estimation were used to classify galaxies as early-, late-types (Coleman et al. 1980) and starbursts (Kinney et al. 1996). A total of 17 were classified as starbursts, 9 as late-types and 2 as early-types. The colors of the starbursts which were reproduced by the stellar evolutionary synthesis code Starburst99 (Leitherer et al. 1999) are typical of a mixed population of bursts with ages $<1$ Gyr. Galaxy morphology was evaluated by measuring the light concentration (C), asymmetry (A) and clumpiness (S) of the ACS images in the rest-frame B band using the method by Conselice (2003). Early-type galaxies are expected to have a high concentration index and low asymmetries whereas late-type (disks) galaxies have low light concentration and high asymmetries. Three objects in the U-band sample have X-ray emission. The point-like object is one of the most powerful CXO sources (Giacconi et al. 2002) in the entire ACS field (Soft X-ray flux $=4.00 \pm .05 \times 10^{-14}$ and Hard X-ray flux $8.06 \pm .17 \times 10^{-14}$ ), it is a Type I AGN. The other two objects are extended and have total X-ray $(0.2-8 \mathrm{keV})$ fluxes $1.77 \times 10^{-16}$ and $2.66 \times 10^{-16}$ erg s${ }^{-1} \mathrm{~cm}^{-2}$ which are three orders of magnitude lower than the Type I AGN $\left(1.15 \times 10^{-13}\right)$. The object with the lowest X-ray flux has a spectral type typical of starbursts and peculiar late-type morphology. The third object has a spectral type similar to intermediate-late spirals.

The majority of the galaxies have lower values of light concentration and higher asymmetries than typical early and late-type galaxies. Based on the morphologies we conclude that near-UV sources at intermediate-z originate in a variety of objects: dwarf ellipticals, early- and late-spirals and peculiar objects which resemble mergers in progress. Intermediate redshifts are an important epoch when the rise in the volume-averaged star formation rate occurs. The population of objects that contributes to this rise is not homogeneous, but made of galaxies of different types experiencing strong star-formation. A gallery of objects are at http://goods.gsfc.nasa.gov/goods/duilia/gallery.

\section{References}

Bertin, E., \& Arnouts, S. 1996, A\&AS, 117, 393

Coleman, G.D., Wu, C-C., \& Wedman, D.W. 1980, ApJS, 43, 393

Conselice, C. 2003, ApJS, 147, 1

Giacconi, R. et al. 2002, ApJS, 139, 369

Giavalisco, M., et al. 2004, ApJ, 600, L93

Kinney, A. et al. 1996, ApJ, 467, 38

Leitherer, C., et al. 1999, ApJS, 123, 3

Madau, P., et al. 1996, MNRAS, 283, 1388

Mobasher, B., et al. 2004, ApJ, 600, L167 